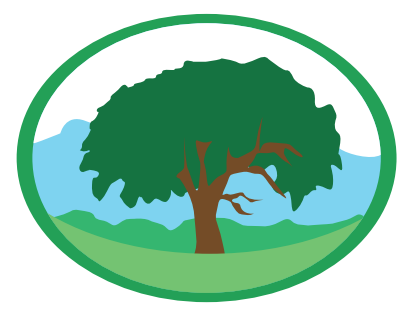

\title{
Metodologia para medição da potência necessária ao corte por um triturador florestal
}

MACHADO, A.L.T. ${ }^{1}$; TIMM, M. W. ${ }^{3}$; MACHADO, R.L.T. ${ }^{1}$; TONUCCI, R. G. ${ }^{4}$; MEDEIROS F. A. ${ }^{2}$

${ }^{1}$ Prof. Dr., Departamento de Engenharia Rural, Faculdade de Agronomia Eliseu Maciel, Universidade Federal de Pelotas, Pesquisador CNPq

${ }^{2}$ Prof. Dr., Departamento de Engenharia Rural, Faculdade de Agronomia Eliseu Maciel, Universidade Federal de Pelotas

${ }^{3}$ Prof. MSc., Instituto Federal Sul-Rio-Grandense.

${ }^{4}$ Pesquisador, Empresa Brasileira de Pesquisa Agropecuária

Palavras-chave: máquinas

agrícolas, exploração

sustentável, semiárido, projeto

de máquina

\begin{abstract}
Resumo
Para execução do processo de raleamento na Caatinga é importante utilizar máquinas do tipo rotores trituradores acoplados em tratores de baixa potência, visando a diminuição de tempo e mão-de-obra dos agricultores familiares na, implantação de sistemas de exploração sustentáveis, sistemas agrossilvipastoris. Para o projeto de uma máquina que seja acessível a estes produtores, o primeiro passo é se conhecer a potência de corte necessária para a operação de raleamento. 0 objetivo deste trabalho foi desenvolver e avaliar uma metodologia de ensaio para medir a potência durante o corte de uma espécie arbórea, podendo assim avaliar os parâmetros a serem utilizados futuramente no projeto da máquina. Como espécie teste, utilizou-se o eucalyptus citriodora, por ser uma madeira que apresenta resistência de corte elevada e similar ao das espécies da Caatinga. Observouse que a metodologia proposta, ao padronizar os procedimentos, permitiu adquirir de forma rápida e precisa os valores dos parâmetros de importância no projeto de máquinas destinadas a cortar e triturar espécies arbóreas. Também a utilização dos dados obtidos por meio da aplicação da metodologia, possibilitam maior confiabilidade e precisão no projeto de máquinas destinadas a este tipo de trabalho.
\end{abstract}

\section{Methodology for obtaining the cutting power of a forest crusher}

Key-words: agricultural machinery, sustainable exploitation, semiarid, machine design

\begin{abstract}
For executions the thinning process in Caatinga is important to use machines as Rotors Crushers coupled in low- power tractors, aimed at decrease of time and hand labor of Family Farmers in the implementation of sustainable exploration systems, agrosylvopastoral Systems. For the project for the design of a machine that is accessible these producers, the first step is knowing the cut power needed for a thinning operation. The objective of this study was to develop and evaluate a test methodology to measure the power during the cutting of a tree species and can evaluate the cutting parameters used in the future in machine design. As a test species, we used the eucalyptus citriodora, as this wood has cut high resistance and similar to Species of Caatinga. It was observed that the methodology proposal, to the standardize procedures, allows to get quickly and accurately the values of importance parameters in the design of machines designed to cut and grind tree species. Also the use of data obtained through the application of the methodology , enable greater reliability and precision in machine design intended for this type of work.
\end{abstract}




\section{INTRODUÇÃO}

O Nordeste Brasileiro abrange uma área de 1,54 milhóes de quilômetros quadrados, o que corresponde a $18 \%$ do território nacional e $28 \%$ da população brasileira. Nesta regiáo, encontra-se o chamado semiárido brasileiro, que segundo IBGE (2006) abriga cerca de 22.200.000 pessoas, sendo considerado um dos semiáridos mais populosos do mundo.

No semiárido a agricultura familiar ainda é praticada de forma itinerante, na maioria das propriedades, utilizando o sistema tradicional de desmatamento e queimada. A maior parte dos agricultores faz o corte raso da Caatinga e a queima da vegetação, cultivando o solo por aproximadamente dois anos e abandonando em seguida devido as reduçóes drásticas na fertilidade e na produção destas áreas. Na pecuária, o sobrepastejo também ocorre na maioria dos sistemas, alterando a estrutura do estrato herbáceo podendo causar a exaustão da vegetação forrageira, levando igualmente à degradação (Pereira Filho, 2013).

Segundo Araujo Filho (2002), a diversificação de atividades executadas no semiárido brasileiro inclui atividades agrícolas, pecuárias e madeireiras. A maneira tradicional de exploração tem sido apontada como um dos principais fatores para a degradação do ambiente e baixa capacidade produtiva das propriedades rurais agrícolas, especialmente as familiares. Uma alternativa a este modelo de exploração vem a ser o chamado sistema agro-florestal (SAF), que integra atividades agrícolas, pecuárias e florestais, tendo surgido como potencial alternativo aos sistemas tradicionalmente utilizados pelos sertanejos (Campanha, 2011).

De acordo com Araujo Filho (2002), os sistemas agroflorestais pecuários desenvolvidos para a Caatinga possuem as seguintes vantagens; redução na prática da agricultura tradicional de desmatamento e queimadas, conservação e manutenção do recurso forrageiro nativo pelo uso de estratégias mais sustentáveis de manejo de pastagens, aumento da produtividade agrícola (grãos. $\mathrm{ha}^{-1}$.ano), pecuária de forma sustentável e com mínimo uso de insumos externos, aumento e estabilidade da oferta de alimentos, como: milho, feijão, mandioca, carne, leite e conservação de forragem e uso de bancos de proteína, reduzindo a estacionalidade da produção de forragem na época seca. Segundo Barreto, (2010), o manejo agroecológico da Caatinga aumenta a capacidade produtiva do solo, diminui o uso de insumos materiais e reduz a emissão de poluentes atmosféricos.

Trata-se, portanto, de uma estratégia para uso sustentável da vegetação local, consistindo em realizar cortes seletivos em espécies de pouco valor forrageiro e madeireiro, reduzindo a densidade destas plantas na área, permitindo assim, que outras espécies, especialmente do estrato herbáceo possam se desenvolver e servir de fonte de alimentação para os animais (Cavalcante, 2013).

Para a execução do SAF se faz necessário o corte de espécies vegetais, raleamento, que vem a ser uma conduta de manipulação da vegetação nativa da Caatinga utilizada para aumentar a oferta de forragem aos rebanhos mantidos nesse tipo de pasto. O raleamento consiste em gerar ruas ( 15 metros) entre faixas de árvores nativas (20 a 30 metros), as quais são todas preservadas. Estas ruas são feitas através de desmatamento das árvores que são cortadas até a raiz (na base das plantas) e os restos são picotados em pequenas partículas. As vantagens deste método está na menor necessidade de mão de obra e na possibilidade de utilização de equipamentos agrícolas que possam auxiliar o agricultor nos tratos culturais e no manejo animal. (Cavalcante, 2013).

Existem diferentes tipos de equipamentos empregados para a execuçáo do raleamento, os quais são acoplados em tratores agrícolas de grande potência, apresentando custo inacessível aos agricultores familiares do semiárido.

Uma alternativa para implantação deste sistema, pelos agricultores familiares do semiárido, vem a ser a de contar com uma máquina capaz de cortar e triturar a vegetação por meio de um rotor, que tenha um preço de aquisição viável e possa ser utilizada por tratores de menor potência. Para o projeto de uma máquina deste tipo o primeiro passo é determinar a demanda de potência de corte da vegetação por meio do rotor triturador.

Segundo a Norma NBR 7190, ABNT (1997), os ensaios para determinação das propriedades das madeiras são de tração, compressão, cisalhamento, fendilhamento, flexão e dureza, entretanto nesta 
Norma não se enquadram ensaios de cortes dinâmicos para medir a potência de corte. Já a Norma D166687, ASTM (1994), refere-se aos testes de usinagem de madeiras, como aplainamento, furação e lixamento, contudo estes ensaios são destinados às fábricas de madeira e visam analisar defeitos em função de parâmetros de corte, não apresentando ensaios sobre esforço de corte ou potência de corte em função de parâmetros como rotação, avanço e ângulo de ataque da ferramenta.

Néri (1999), ensaiou corpos de prova de três espécies de eucalipto analisando a força paralela de corte em função do ângulo de ataque da ferramenta de corte e a espessura do cavaco, entretanto todos os ensaios foram realizados com velocidades de corte baixas.

Souza (2011), verificou a influência dos parâmetros de usinagem no consumo de energia específica de corte da madeira dos clones de Eucalyptus sp, numa serra circular, e obteve menor consumo de energia interagindo os parâmetros de corte, como velocidade de corte, avanço e número de dentes.

Segundo McKenzie (1960), existem dois tipos básicos de corte na madeira, o ortogonal e o periférico. No corte ortogonal o fio de corte da ferramenta é perpendicular à direção de movimento da peça de madeira, sendo definido por meio da utilização de dois numerais, onde o primeiro é ângulo entre a aresta principal da ferramenta de corte e as fibras da madeira e o segundo o ângulo entre a direção de corte e as fibras da madeira. Desta forma definem-se três tipos de corte $90-0,90-90$ e $0-90$. No corte periférico a ferramenta de corte é instalada em um cabeçote rotativo, a fim de se obter um mesmo cilindro de corte. As operaçôes de aplainamento, fresamento e torneamento são exemplos de corte periféricos, mas são situaçóes de trabalhos bem próximas de um corte ortogonal.

Devido não existir metodologia de ensaio para avaliação da potência necessária ao corte de amostras de madeira, por mecanismos do tipo rotor triturador, o objetivo deste trabalho foi o de desenvolver uma metodologia de ensaio com esta finalidade. A fim de se ratificar os procedimentos metodológicos aqui propostos simulou-se a rotação do mecanismo triturador, ao mesmo tempo em que se executou a variação da velocidade de deslocamento da máquina e ângulo de ataque da ferramenta responsável pelo corte da espécie vegetal.

Tal procedimento se fez necessário para obtenção da força exigida ao corte da espécie arbórea. Desta forma torna-se possível a escolha e definição do tipo de material a ser utilizado no projeto da ferramenta de corte, a rotação mais adequada a execução do trabalho e por via de consequência a potência necessária ao trator no qual a máquina será acoplada.

\section{MATERIAL E MÉTODOS}

$\mathrm{Na}$ execução dos ensaios para definição da metodologia utilizou-se como espécie vegetal de teste o Eucalipto Citriodora. Esta foi selecionada com o objetivo de ensaiar um tipo de madeira com padrão de resistência ao corte semelhante aquelas existentes na Caatinga.

Para simular o efeito giratório e de avanço do triturador foi usada uma fresadora universal, marca TOS, modelo Fresadora Vertical, figura 1, provida de mesas com movimentos vertical, transversal e longitudinal e medidor de potência analógico.

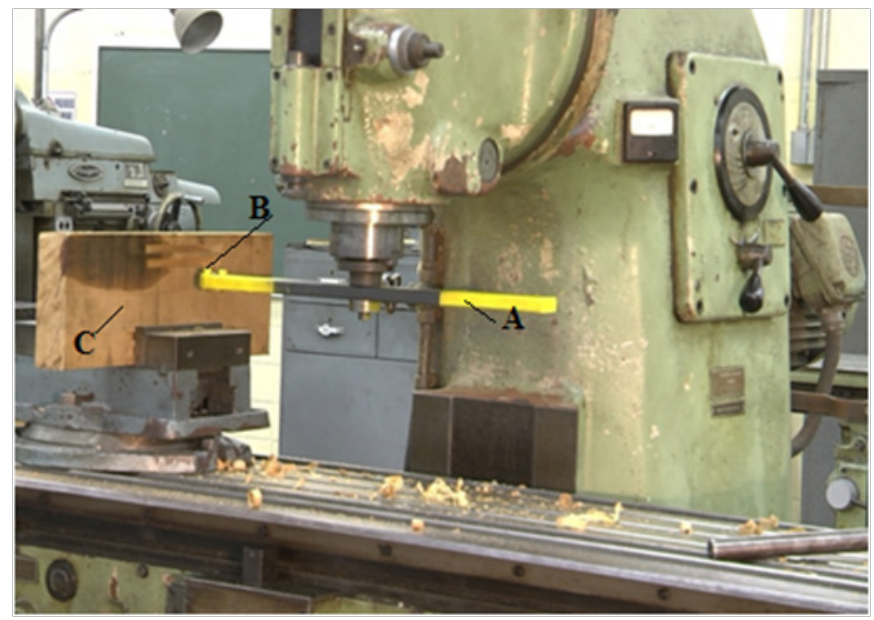

Figura 1. Fresadora, onde: A. porta-ferramentas. B. ferramenta. C. amostra de madeira fixada na mesa longitudinal.

Como a fresadora não conta com instrumentação eletrônica para a aquisição dos dados oriundos do medidor de potência da mesma, o qual é analógico, para o registro dos mesmos foi utilizada uma filmadora digital marca Sony, modelo Hxr.

No sentido de se obter a variação do ângulo e diâmetro de corte da ferramenta construiu-se um 
porta ferramentas, figura $1 \mathrm{~A}$ e figura $2 \mathrm{~B}$, de maneira que fosse possível a obtenção de variação no ângulo de ataque da ferramenta com um diâmetro de corte similar ao daquelas que são fixadas em rotores trituradores comerciais (DENIS CIMAF INC., 1991).

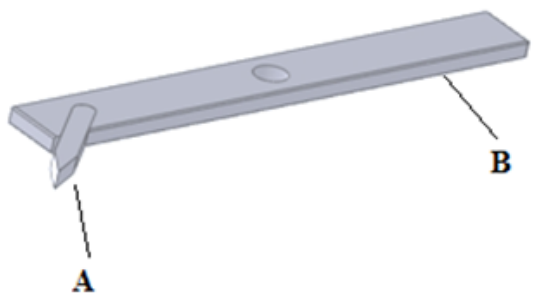

Figura 2. Porta ferramentas. Onde: A. ponteira de corte. B. Haste porta ferramenta

Devido ao fato de que os rotores trituradores normalmente cortam as árvores na direção de corte longitudinal, DENIS CIMAF INC. (1991), optou-se por utilizar o corte do tipo periférico no sentido das fibras, ou seja, na direção 90 - 0, conforme Woodson (1960).

Como corpos de prova foram utilizados cinco espécimes do mesmo material, Eucalipto Citriodora, medindo $80 \times 160 \times 500 \mathrm{~mm}$ tendo-se executado quatro cortes em cada face maior do corpo de prova.

Como ferramenta de corte, figura 2A, optou-se pela utilizaçáo de uma ponteira de aço rápido com $10 \%$ de Cobalto, a qual é utilizada na usinagem de aços. Como ângulo de ataque desta ferramenta utilizou-se $30^{\circ}$ e $45^{\circ}$.

A fim de simular as possíveis rotaçôes da ferramenta de corte utilizaram-se no desenvolvimento desta metodologia as seguintes rotaçóes: 90, 180, 335, 710 e $1400 \mathrm{rpm}$.

A velocidade linear de avanço da mesa da fresadora, em $\mathrm{mm} \mathrm{min}^{-1}$, onde se fixou o corpo de prova, foi usada para simular a velocidade de trabalho da máquina em direção à espécie arbórea. Desta forma utilizaram-se como velocidades de avanço: 80, 112, 160 e $224 \mathrm{~mm}$ $\min ^{-1}\left(0,0048 \mathrm{~km} \mathrm{~h}^{-1}, 0,0067 \mathrm{~km} \mathrm{~h}^{-1}, 0,096 \mathrm{~km} \mathrm{~h}^{-1}\right.$, $\left.0,013 \mathrm{~km} \mathrm{~h}^{-1}\right)$.

A potência necessária ao corte do corpo de prova foi lida no medidor acoplado a fresadora. Para cada rotação mediu-se a potência da máquina em vazio, ou seja, sem a máquina usinar (somente girando), sendo que para a mesma rotaçáo fez-se a leitura da potência de corte da máquina usinando a madeira.
Este procedimento foi executado para uma dada rotação variando-se o avanço. Posteriormente mudouse a rotação e foram medidas as potências para toda faixa de avanço novamente. $\mathrm{O}$ processo foi repetido em todas as rotaçôes até a última de $1400 \mathrm{rpm}$ e para os dois ângulos de ataque da ferramenta, ou seja, $30^{\circ}$ e $45^{\circ}$.

\section{RESULDADOS E DISCUSSÃO}

Os valores apresentados nas Tabelas 1e 2 referem-se à potência consumida pela fresadora durante o corte, para dois ângulos de ataque e quatro velocidades de avanço. Mediu-se a potência da máquina quando da realização do corte da madeira e em vazio, por meio da diferença obteve-se a potência de corte real da madeira. Tal procedimento foi adotado no sentido de se verificar a possibilidade de simulaçáo do corte realizado por máquinas que utilizam mecanismos rotativos para triturar madeira.

Verificou-se que a medição da potência necessária ao corte foi obtida de forma rápida e precisa indicando que a metodologia aqui proposta pode ser utilizada na simulação deste tipo de ensaio. Tal fato revestese de importância visto que desta forma tem-se uma importante ferramenta para testes de resistência ao corte de amostras de madeira a qual possibilita a obtenção das bases teóricas para o projeto deste tipo de equipamento, o que até o momento inexistia.

Tabela 1. Valores médios da Potência de corte dos corpos de prova (P) em kW, em função da rotação (n) em $\mathrm{rpm}$, para ângulo de ataque da ferramenta $(\gamma)$ igual a $30^{\circ}$ em diferentes velocidades de avanço (v) em $\mathrm{mm} \mathrm{min}^{-1}$.

\begin{tabular}{rllll}
\multicolumn{1}{l}{$\mathrm{n}$} & $\mathrm{v}=80$ & $\mathrm{v}=112$ & $\mathrm{v}=160$ & $\mathrm{v}=224$ \\
\hline 90 & 0,05 & 0,25 & 0,45 & 1,00 \\
180 & 0,11 & 0,11 & 0,15 & 0,30 \\
335 & 0,35 & 0,40 & 0,90 & 1,00 \\
710 & 0,11 & 0,20 & 0,23 & 0,28 \\
1400 & 0,30 & 0,55 & 1,00 & 1,15 \\
\hline
\end{tabular}

A análise dos dados constantes das tabelas 1 e 2 permite inferir ainda que a potência necessária ao corte do corpo de prova é crescente conforme aumenta a rotação de trabalho da ferramenta, independentemente da velocidade de avanço. Exceção feita para a rotação de $710 \mathrm{rpm}$ na qual, independentemente da velocidade e 
Tabela 2. Valores médios da Potência de corte dos corpos de prova (P) em kW, em função da rotação (n) em rpm, para ângulo de ataque da ferramenta $(\gamma)$ igual a $45^{\circ}$ e velocidade de avanço ( $\mathrm{v}$ ) em $\mathrm{mm} \mathrm{min}^{-1}$.

\begin{tabular}{rllll}
\multicolumn{1}{l}{$\mathrm{n}$} & $\mathrm{v}=80$ & $\mathrm{v}=112$ & $\mathrm{v}=160$ & $\mathrm{v}=224$ \\
\hline 90 & 0,10 & 0,10 & 0,20 & 0,22 \\
180 & 0,08 & 0,10 & 0,24 & 0,29 \\
335 & 0,20 & 0,60 & 0,95 & 1,58 \\
710 & 0,16 & 0,20 & 0,31 & 0,39 \\
1400 & 0,50 & 0,70 & 1,25 & 1,27 \\
\hline
\end{tabular}

ângulo de ataque da ferramenta, a potência necessária ao corte do corpo de prova se reduz. Era esperado que a potência necessária ao corte do corpo de prova fosse crescente com o aumento da rotação da ferramenta, fato que não ocorreu e pode ser identificado pela aplicação do método aqui proposto.

As informaçóes obtidas e relatadas demonstram a importância de se contar com uma metodologia padrão para este procedimento, pois através da aplicação da metodologia aqui proposta foi possível além da identificação da potência necessária ao corte da espécime arbórea, objetivo primário deste trabalho, também a observação de situações diferenciadas, as quais necessitam de estudos mais aprofundados a fim de se verificar o porque da ocorrência do fenômeno.

Outra informaçáo importante conseguida com a aplicação da metodologia é a possibilidade de montarse um gráfico correlacionando-se a potência de corte com a velocidade de avanço do equipamento, para exemplificar tal fato montou-se a figura 3 , onde se tem a situação em que se obteve a menor potência necessária ao corte, ou seja, ferramenta com ângulo de ataque de $30^{\circ}$, velocidade de avanço de $221 \mathrm{~mm} \mathrm{~min}^{-1}$ para a rotação de $710 \mathrm{rpm}$.

Desta forma por meio da aplicação da metodologia e dos dados obtidos, além da montagem da figura 3, foi possível ainda obter-se uma curva de tendência de comportamento dos dados gerados, que no presente caso foi uma relação linear, a qual é descrita pela equação $\mathrm{y}=0,001 \mathrm{x}+0,031$, onde $\mathrm{y}$ representa a potência e x o avanço, com $\mathrm{r}^{2}=0,97$, significando que tal equação representa $97 \%$ dos dados. A utilização de equaçôes deste tipo, com um adequado grau de precisão, permite a análise da potência de corte em velocidades diferentes daquelas que foram testadas, possibilitando a utilizaçáo destes dados no projeto das máquinas.

Figura 3. Potência de corte em relação a velocidade de avanço, para a rotação de 710 rpm e ângulo de ataque de $30^{\circ}$.

\section{CONCLUSÕES}

A utilização da metodologia aqui proposta possibilitou padronizar os procedimentos para obtenção da potência de corte em corpos de prova de espécimes arbóreas;

A metodologia permitiu a obtenção, de forma rápida e precisa, dos fatores que são importantes no dimensionamento de máquinas destinadas a triturar espécimes arbóreas;

A utilização dos dados, por meio da metodologia proposta, possibilita maior confiabilidade e precisão no projeto de máquinas destinadas ao corte e trituração de espécimes arbóreas.

\section{LITERATURA CITADA}

AMERICAN SOCIETY FOR TESTING AND MATERIALS. ASTM D 1666- 87: standard method for conducting machining tests of wood and wood base materials (reapproved 1994). Philaldelphia, 1995. p.226-245.

ARAÚJO FILHO, J. A.; CARVALHO, F. C.; GARCIA, R.; SOUSA, R. A. Efeitos da manipulaçáo da vegetaçáo lenhosa sobre a produçáo e compartimentalizaçáo da fitomassa pastável de uma caatinga sucessional. Revista Brasileira de Zootecnia, v.31, n.1, p.11-19, 2002.

ASSOCIAÇĀO BRASILEIRA DE NORMAS TÉCNICAS. Projeto de estruturas de madeira ABNT (NBR-7190). Rio de Janeiro. 1997, 107p.

BARRETO, H. F. M.; SOARES, J. P. G.; MORAES, D. A. E. F.; SILVA, A. C. C.; SALMAN, A. K. D. Impactos ambientais do manejo agroecológico da caatinga no Rio Grande do Norte. Pesquisa agropecuária brasileira, vol.45, n¹0, p.1073-1081, 2010.

CAMPANHA, M. M.; ARAÚJO, F. S.; MENEZES, M. O. T.; SILVA, V. M. A.; MEDEIROS, H. R. Estrutura da comunidade vegetal arbóreo-arbustiva de um sistema agrossilvipastoril, em Sobral-CE. Revista Caatinga, v.24, p.94-101, 2011.

CAVALCANTE, A. C. R.; FERNADES, F. E. P.; TONUCCI, R. G.; SOLVA, N. L.. Tecnologias para o uso sustentável da Caatinga. Campina Grande: EPGRAF, 2013, p.95-112.

DENIS CIMAF INC. Desbastadores Florestais. Canadá-1991. Disponível em <HTTP://deniscimaf.com. Acesso em: 19 Ago 2015.

NÉRI, A.C; GONÇALVEZ, R.; HERNADEZ, R. E. Forças de corte ortogonal 90-0 em três espécies de madeira de Eucalipto. Revista Brasileira de Engenharia Agrícola e Ambiental, v3, n.2, p.239-244, 1999.

NÉRI, A.C; GONÇALVEZ, R.; HERNADEZ, R. E. Forças de corte ortogonal 90-90 em três espécies de madeira de 
Eucalipto. Revista Brasileira de Engenharia Agrícola e Ambiental, v4, n.2, p.275-280, 2000.

McKENZIE, W.M;. Fundamental aspects of the wood cutting process. Forest Products Journal, v.10, n.9, p.447-456, 1960.

PEREIRA FILHO, J. M.; SILVA, A. M. A; CÉZAR, M. F. Manejo da caatinga para produçáo de caprinos e ovinos. Revista Brasileira de Saúde e Produção Animal, v.14, n.1, p.7790, 2013
SOUZA, E. M.; SILVA, J. R. M.; LIMA, J. T.; NAPOLI, A.; RAAD, T. J.; GONTIJO, T. G. Energia específica de corte em serra circular para os clones de eucalyptus vm01 e Mn 463. Revista Cerne, v.17, n.1, p. 09-115, 2011.

WOODSON, G. E.; KOCH, P. Tool forces and chip formation in orthogonal cutting of loblolly pine. Research Paper SO - 52. U.S. Department of Agriculture, Forest Service, 1960. 